\title{
LISA INTERFEROMETER SENSITIVITY TO SPACECRAFT MOTION
}

\author{
Michael Peterseim ${ }^{1,2}$, D.I. Robertson ${ }^{2}$, K. Danzmann ${ }^{2}$, H. Welling ${ }^{1}$ and P. Bender ${ }^{3}$ \\ ${ }^{1}$ Laser Zentrum Hannover e.V., Hollerithallee 8, D-30419 Hannover, Germany \\ ${ }^{2}$ Max-Planck-Institut für Quantenoptik, Callinstraße 38, D-30167 Hannover, Germany \\ ${ }^{3}$ Joint Institute for Laboratory Astrophysics, Boulder, Colorado, USA
}

\begin{abstract}
A study has been performed to assess the sensitivity of the LISA interferometer to motion of the spacecraft (S/C) with respect to the proof mass. The results are presented as a functions of arm length and Fourier frequency. In addition different optical arrangements have been analysed and their effects on the cancellation of the spacecraft motion is discussed.

(C) 2000 COSPAR. Published by Elsevier Science Ltd.
\end{abstract}

\section{ANALYSIS OF INTERFEROMETER RESPONSE TO S/C MOTION}

The approach used in this study, starting with the optical arrangement of the current LISA baseline as outlined by Danzmann et al. (1997 and 1998), was to calculate the phase information detected with the individual photodiodes mounted onto the different optical benches. Methods were defined to extract the gravitational wave signal contained in this phase information by removing laser phase noise as well as noise due to the $\mathrm{S} / \mathrm{C}$ motion. In this section the phase information recorded on the photodiodes is described. The following sections deal with the different data acquisition and data analysis methods, including signal extraction algorithms and possible modifications to the current optical arrangement.

Basic Arrangements and Requirements

Figure 1 shows a schematic diagram of the layout of the LISA interferometer. The arms of the interferometer are defined between the proof masses, e.g. arm 1 is between proof mass A1 and proof mass C2. For simplicity we will just regard the interferometer formed by arm 1 and arm 2 and we will take the far ends as perfect mirrors that introduce no additional phase shift to the beams.

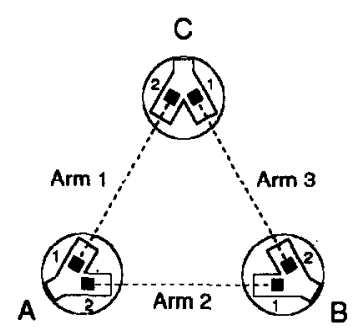

Figure 1 Schematic layout of the LISA interferometer

The proof masses in the local $\mathrm{S} / \mathrm{C}$ are assumed to be rigidly fixed in the inertial frame of the far end mirrors, and the motion of the $\mathrm{S} / \mathrm{C}$ is defined with respect to that frame. The optical arrangement and payload design, as given in Danzmann et al. (1997 and 1998) is shown schematically in Figure 2. On both of the optical benches light from the local laser is sent as a phase reference to diode PD1 and PD2 for Laser L1 and to diode PD3 and PD4 for Laser L2 (cp. figure 2). These diodes are assumed to feed into perfect phase meters with an output $P D(t)=$ Phase $_{\text {remote }}(\mathrm{t})-$ Phase $_{\text {local ref }}(\mathrm{t})$. 
The laser associated with the optical bench $\mathrm{A} 1$ will serve as the master for the whole system and will be locked to an on board reference cavity. The other laser is phase locked to laser 1. Infinite gain and bandwidth of the phaselock are always assumed. For both optical benches the incoming light from the far $\mathrm{S} / \mathrm{C}$ is reflected off the proof mass and superimposed with the local laser on PD1 or PD4 respectively.

A fraction of the light from each laser is reflected off the back of the proof mass and sent to the other optical bench for phase comparison using an optical fibre. Phase noise introduced by this fibre is neglected throughout this work, as it cancels out when the difference of PD2 and PD3 is taken.

The S/C motion under consideration is the residual motion of the satellite surrounding the proof mass that is not corrected by the the drag free control system. The lasers, mirrors and photodiodes are rigidly fixed to the S/C. The "amplitude" of that motion has a maximum tolerable spectral density (Danzmann et al., 1998) of $\widetilde{\delta S}_{\mathrm{s} / \mathrm{c}}=1 \times 10^{-9} \mathrm{~m} / \sqrt{\mathrm{Hz}}$.

The requirement on the applied technique for removing the effect of the residual $\mathrm{S} / \mathrm{C}$ motion is set by the desired overall sensitivity of the instrument. Following the LISA PrePhase-A report (Danzmann et al., 1998), the additional phase error due to that error source has to be significantly less than the envisaged total opticalpath noise budget of $\widetilde{\delta S}_{\mathrm{OP}}=40 \times 10^{-12} \mathrm{~m} / \sqrt{\mathrm{Hz}}$ for frequencies of $1 \mathrm{mHz}$ to $1 \mathrm{~Hz}$.

\section{$\underline{\text { Response of Photodiodes }}$}

We begin with the optical configuration described above and, for simplicity, with the assumption that neither laser noise nor a gravitational wave signal is modulated onto the laser beam. If the system at $t_{0}$ undergoes a step motion $\delta s$ of the $S / C$ relative to the proof masses, as indicated in Figure 2 , the following signals are present on the 4 photodiodes (compare Figure 3 )

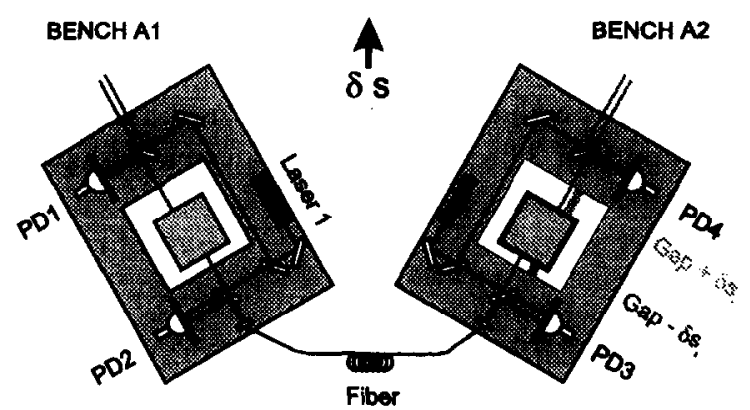

Figure 2 Diagram of the optical Benches.

- PD1: Additional phase of the remote signal of $\delta x_{1}=\nu \delta s_{1} / c$ ( $\nu$ : laser frequency) during the light travel time in arm 1 from $t_{0}$ on ( $\delta s_{1}$ : component of $\delta s$ along the sensitive axis of proof mass 1$)$.

- PD2: Additional phase of the remote signal of $-2 \times \delta x_{2}$ from $t_{0}$ on.

- PD3: Additional phase of the remote signal of $-2 \times \delta x_{1}$ from $t_{0}$ on.

- PD4: Additional phase of the remote signal of $\nu \delta s_{2} / c$ during the light travel time in arm 2 from $t_{0}$ on.

If only diodes PD1 and PD4 were used, the maximum allowed spectral density $\widetilde{\delta S}_{\mathrm{s} / \mathrm{c}}$ would lead to a contribution to the optical-path noise budged 25 times larger than the envisaged overall sensitivity. Therefore a correction scheme has to be applied.

As can be seen, the signals on PD2 and PD3 could in principle be used to remove the phase response to the $\mathrm{S} / \mathrm{C}$ motion by forming the signals

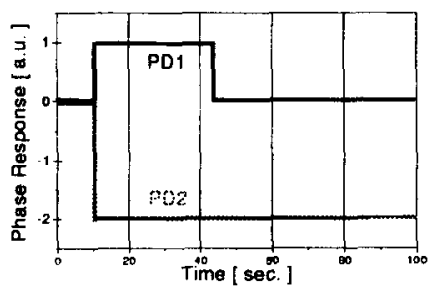

Figure 3 Response of Photodiodes on bench A1. 


$$
S_{1}(t)=P D 1(t)+\frac{1}{2}\left[P D 3(t)-P D 3\left(t-T_{1}\right)\right] \quad S_{2}(t)=P D 4(t)+\frac{1}{2}\left[P D 2(t)-P D 2\left(t-T_{2}\right)\right]
$$

where $T_{i}$ denotes the light travel time in the $i_{\mathrm{th}}$ arm (appr. 33sec.).

The situation is a little more difficult when taking into account laser phase noise and frequency drift. The general beat signal, including laser noise and gravitational wave effects, read on the $i_{\text {th }}$ photodiode is:

$$
\begin{aligned}
& P D 1(t)=\nu_{1} T_{1}+g w_{1}(t)+p_{1}\left(t-T_{1}\right)-p_{1}(t)+\delta x_{1}(t)-\delta x_{1}\left(t-T_{1}\right) \\
& P D 2(t)=\Delta \omega t-p_{1}(t)+p_{2}(t)-2 \delta x_{2}(t) \\
& P D 2(t)=-\Delta \omega t+p_{1}(t)-p_{2}(t)-2 \delta x_{1}(t) \\
& P D 4(t)=\nu_{2} T_{2}+g w_{2}(t)+p_{2}\left(t-T_{2}\right)-p_{2}(t)+\delta x_{2}(t)-\delta x_{2}\left(t-T_{2}\right)
\end{aligned}
$$

Here $p_{i}$ and $g w_{i}$ are the phase noise of laser $i$ and the phase modulation of interferometer arm $i$ due the gravitational wave. The term $\Delta \omega$ represents the frequency difference between the lasers, which can be very large (up to $150 \mathrm{GHz}$ ) and will not even be constant. This makes the phaselock between the two lasers absolutely necessary!

One can either use the signal from diode PD2 or from diode PD3 or a linear combination of those for the feedback loop. The current LISA baseline forsees that laser L1 is locked to a Fabry-Perot interferometer to improve short term stability and laser L2 is locked to the difference of the signals on PD2 and PD3 (Danzmann et al, 1998). As infinite gain and bandwidth are assumed, the phase of laser L2 is controlled to be $P D 2(t)=-\Delta \omega t+p_{1}(t)-\delta x_{1}(t)+\delta x_{2}(t)$. The difference of the signals read on PD2 and PD3 is zero and the other diodes give:

$$
\begin{aligned}
& P D 1(t)=\nu_{1} T_{1}+g w_{1}(t)+p_{1}\left(t-T_{1}\right)-p_{1}(t)+\delta x_{1}(t)-\delta x_{1}\left(t-T_{1}\right) \\
& P D 2(t)=-\left[\delta x_{1}(t)+\delta x_{2}(t)\right]
\end{aligned}
$$

with similar expressions for the signals $P D 3(t)$ and $P D 4(t)$.

For the case of an equal arm interferometer, the $\mathrm{S} / \mathrm{C}$ motion now cancels out when the difference of the signals from PD1 and PD4 is evaluated. A signal can be defined that would be noise-free for perfectly known light travel times $T_{1}=T_{2}$ : Signal $(t)=P D 1(t)-P D 4(t)$.

\section{PERFORMANCE AND ALTERNATIVE OPTICAL ARRANGEMENTS}

\section{Performance of current LISA baseline}

One should note from Eq. 3 that the error does not cancel out if the arms are not equal, not even if the arm lengths are perfectly known. We will now discuss the limitations placed on this procedure by an arm length difference and an error in the knowledge of the actual travel times.

In Fourier space Eq. 3, including the uncertainties in knowledge of armlength $\delta T_{i}$ and the shot noise contributions on the diodes $n_{i}$, reads:

$$
\begin{aligned}
& \widetilde{P D} 1(f)=\widetilde{g w_{1}}(f)+\widetilde{n_{1}}(f)-\widetilde{p_{1}}(f)\left[1-e^{-i \omega\left(T_{1}+\delta T_{1}\right)}\right]+\widetilde{\delta x_{1}}(f)\left[1-e^{-i \omega\left(T_{1}+\delta T_{1}\right)}\right] \\
& \widetilde{P D} 2(f)=-\left[\widetilde{\delta x_{1}}(f)+\widetilde{\delta x_{2}}(f)\right]+\widetilde{n_{2}}(f)
\end{aligned}
$$

with similar expressions for the signals $\widetilde{P D} 3(f)$ and $\widetilde{P D} 4(f)$.

Faller and Giampieri et al. have developed a noise reduction scheme that can be used to remove the laser phase noise from the signals detected on diodes PD1 and PD4 (Faller and Giampieri et al., 1996 and references in it). As the contribution due to the $\mathrm{S} / \mathrm{C}$ motion enters the phase signal in the same way as the laser noise, 
this correction scheme will reduce both of them is the same way. Following the work of Giampieri et al., we get for the difference signal in the long wavelength limit $\left(f T_{1}, f T_{2} \ll 1\right)$ :

$$
\operatorname{Signal}(f) \approx 4 \pi i f \widetilde{N}_{1}(f)\left[\frac{T_{1} \delta T_{2}-T_{2} \delta T_{1}}{T_{1}}\right]+\frac{T_{1} \widetilde{n_{2}}(f)-T_{2} \widetilde{n_{1}}(f)}{T_{1}}+\left[\widetilde{g w_{1}}(f)-\widetilde{g w}_{2}(f)\right]\left[\frac{T_{1}+T_{2}}{T_{1}}\right]
$$

where $\widetilde{N_{1}}(f)=\widetilde{p_{1}}(f)-\widetilde{\delta x_{1}}(f)$.

Eq. 5 gives a requirement on the accuracy with which the armlengths must be determined. As the phase error due to the laser noise as taken from Danzmann (1998) is at least three orders of magnitude larger than the contribution due to the $\mathrm{S} / \mathrm{C}$ motion, the requirement on the accuracy is totally determined by the laser phase noise. The work of Giampieri et al. (1996) showed that for reasonable values of $\delta T_{1}-\delta T_{2}=200 \mathrm{~m} / \mathrm{c}$ and a fractional difference in arm length of $1 \%$ the term due to laser phase noise and $\mathrm{S} / \mathrm{C}$ movement noise can be reduced to well below the level needed for measuring the optical path differences over the frequency range of interest.

\section{Alternative optical arrangements: direct phaselock}

So far, the photodiodes have been assumed to feed into perfect phase meters. For the main signal $P D 1$, the high frequency laser phase noise will be low enough so that phase measurements a few hundred times per second followed by digital filtering would be adequate to avoid aliasing in the high frequency noise. However, it currently is assumed that laser 2 would not be locked to a cavity, so its phase noise level would be substantially higher than for laser 1 . Thus the phase meters would have to operate at a considerably higher rate if the linear conbination of signals PD2 - PD3 is used to phase lock laser 2.

As an alternative, it appears useful to consider an optical arrangement where laser 2 is locked to the output of a single photodiode without a phase meter following it. This could be done in several ways, each requiring at least one extra photodiode and an additional step in processing the data. However, avoiding locking to the difference of two phase meter outputs seems to be a significant simplification.

One approach is not to use one of the four above mentioned diodes for the phaselock of the two lasers. A tiny amount of light can be sent both ways between laser 1 and laser 2 by an extra fiber, and the beat signals detected by two additional photodiodes. One of these diode signals is used to phase lock laser 2 . The signals on the other diodes are in Fourier space (again assuming infinite gain and bandwidth of the phaselock):

$$
\begin{aligned}
& \widetilde{P D} 1(f)=\widetilde{g w_{1}}(f)-\widetilde{p_{1}}(f)\left[1-e^{-i \omega\left(T_{1}+\delta T_{1}\right)}\right]+\widetilde{\delta x_{1}}(f)\left[1-e^{-i \omega\left(T_{1}+\delta T_{1}\right)}\right] \\
& \widetilde{P D} 2(f)=-2 \widetilde{\delta x_{2}}(f)
\end{aligned}
$$

with similar expressions for the signals $\widetilde{P D} 3(f)$ and $\widetilde{P D} 4(f)$.

Now the information about the displacement between proof mass one and the $\mathrm{S} / \mathrm{C}$ and between proof mass 2 and the $\mathrm{S} / \mathrm{C}$ is directly accessible. Therefore the $\mathrm{S} / \mathrm{C}$ motion can be removed from the signals on PD1 and on PD4 independently.

$$
\begin{aligned}
\widetilde{P} 1(f) & =\widetilde{P D} 1(f)+\frac{1}{2} \widetilde{P D} 3(f)\left[1-e^{-i \omega\left(T_{1}+\delta T_{1}\right)}\right] \\
& =\widetilde{g w_{1}}(f)-\widetilde{p_{1}}(f)\left[1-e^{-i \omega\left(T_{1}+\delta T_{1}\right)}\right]+\widetilde{\delta x_{1}}(f) e^{-i \omega T_{1}}\left[1-e^{-i \omega \delta T_{1}}\right]
\end{aligned}
$$

Forming the equivalent signal for PD4 and taking the difference, one gets the gravitational wave signal and the laser phase noise plus the reduced $\mathrm{S} / \mathrm{C}$ movement noise contribution, that is shown in Figure 4 for a typical arm length error of $\delta T_{i}=20 \mathrm{~km} / \mathrm{c}$. 


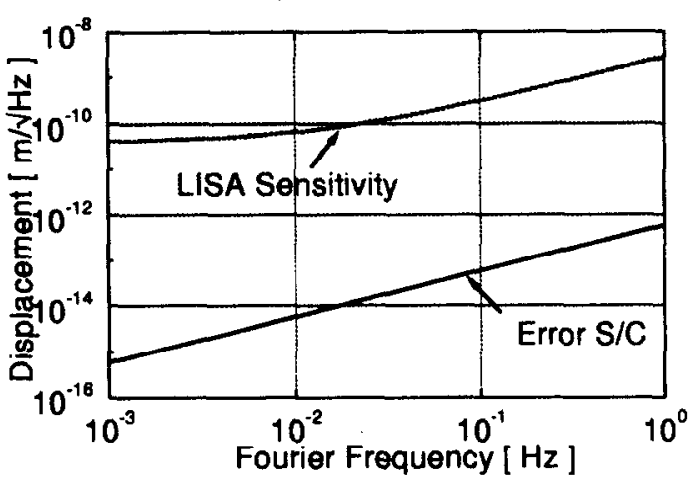

Figure 4 Total optical-path noise budget of the LISA interferometer (LISA Sensitivity) and additional error contributions due to $S / C$ movement for the case of direct phase lock (Error $S / C$ ).

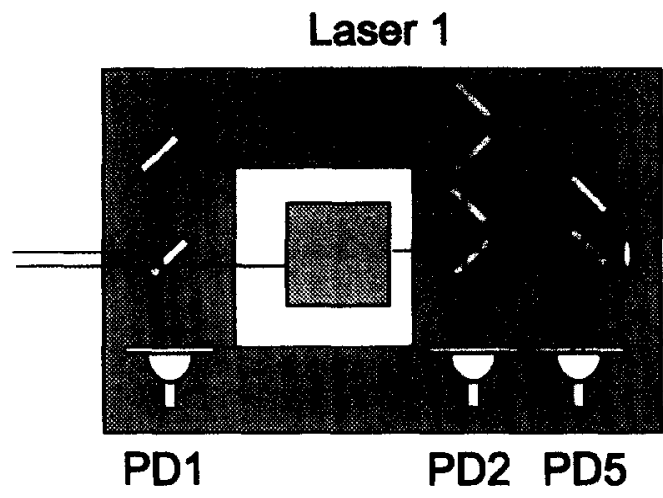

Figure 5 Alternative arrangement of the optical bench, introducing a dedicated diode for $S / C$ movement readout. This diode detects only $\widetilde{\delta s_{1}}(f)$ without any possible time dependent delay due to a fibre

Another approach to access the information about the displacement between proof masses and the $\mathrm{S} / \mathrm{C}$ is to read out this displacement interferometrically using a dedicated diode. For that purpose additional diodes can be placed on the optical benches as shown in Figure 5. These diodes, if appropriately arranged, detect only $\widehat{\delta x_{1}}(f)$ or $\widetilde{\delta x_{2}}(f)$ respectively, without any possible time dependent delay due to a fibre. Now one can continue as in Eq. (8), assuming that PD3 is used for the phase locking of laser 2.

\section{CONCLUSIONS}

This study has shown several ways in which the residual displacement error of $\widetilde{\delta S}_{\mathrm{s} / \mathrm{c}}=10^{-9} \mathrm{~m} / \sqrt{\mathrm{Hz}}$ can be prevented from producing a substantial phase error of the interferometer readout. The current LISA baseline scheme overcomes the problem by bouncing some light off the back of the proof masses. By using the phase information carried by this light for the phaselock of the two lasers at the same $\mathrm{S} / \mathrm{C}$, the usual noise reduction scheme reduces the $\mathrm{S} / \mathrm{C}$ noise contribution well below the envisaged sensitivity of the LISA interferometer.

Two alternative optical arrangements have been proposed to reduce the additional error due to the $\mathrm{S} / \mathrm{C}$ to a tolerable level even before the noise reduction scheme is applied. These arrangements introduce one or two additional photodiodes to either perform a direct phaselock, or measure directly the displacement between $\mathrm{S} / \mathrm{C}$ and proof mass interferometrically.

\section{ACKNOWLEDGEMENTS}

This work was supported by the European Space Agency under Grant ESTEC/12658/NL/MV. We would like to thank the Wernher von Braun - Stiftung zur Förderung der Weltraumwissenschaften, Schopfheimer Straße 16 in Berlin for support.

\section{REFERENCES}

Danzmann, K. et al., LISA Pre-Phase A Report, $2^{\text {nd }}$ Edition, MPQ 233, July 1998

Danzmann, K. et al., LISA Payload Definition Document, Version 03 23/4/97

JPL Advanced Projects Design Team, LISA - Final report, JPL D-14350 24/2/97

Giampieri et al., Algorithms for Unequal-Arm Michelson Interferometers, Optics Communications 123 (1996) 669-678 